\title{
Retinopathy Induced by Zinc Oxide Nanoparticles in Rats Assessed by Micro-computed Tomography and Histopathology
}

\author{
Young Hee Kim', Kyung A Kwak', Tae Sung Kim², Ji Hyeon Seok², Hang Sik Roh², Jong-Kwon Lee², \\ Jayoung Jeong ${ }^{2}$, Eun Ho Meang ${ }^{3}$, Jeong-sup Hong ${ }^{3}$, Yun Seok Lee ${ }^{4}$ and Jin Seok Kang ${ }^{1}$ \\ ${ }^{1}$ Department of Biomedical Laboratory Science, Namseoul University, Cheonan, Korea \\ ${ }^{2}$ Toxicological Research Division, National Institute of Food and Drug Safety Evaluation, Ministry of Food and Drug Safety, \\ Osong Health Technology Administration Complex, Cheongwon, Korea \\ ${ }^{3}$ Health Care Research Laboratory, Korea Testing and Research Institute, Seoul, Korea \\ ${ }^{4}$ Department of Health Administration, Namseoul University, Cheonan, Korea
}

(Received April 23, 2015; Revised May 25, 2015; Accepted June 8, 2015)

\begin{abstract}
Nanotechnology has advanced at an extremely rapid pace over the past several years in numerous fields of research. However, the uptake of nanoparticles (NPs) into the body after administration through various routes may pose a risk to human health. In this study, we investigated the potential ocular toxicity of 20$\mathrm{nm}$, negatively- charged zinc oxide ( $\mathrm{ZnO}) \mathrm{NPs}$ in rats using micro-computed tomography (micro-CT) and histopathological assessment. Animals were divided into four groups as control group, ZnO NPs treatment group (500 mg/kg/day), control recovery group, and ZnO NPs treatment and recovery group. Ocular samples were prepared from animals treated for 90 days (10 males and 10 females, respectively) and from recovery animals ( 5 males and 5 females, respectively) sacrificed at 14 days after final treatment and were compared to age-matched control animals. Micro-CT analyses represented the deposition and distribution of foreign materials in the eyes of rats treated with ZnO NPs, whereas control animals showed no such findings. X-ray fluorescence spectrometry and energy dispersive spectrometry showed the intraocular foreign materials as zinc in treated rats, whereas control animals showed no zinc signal. Histopathological examination revealed the retinopathy in the eyes of rats treated with ZnO NPs. Neuronal nuclei expression was decreased in neurons of the ganglion cell layer of animals treated with ZnO NPs compared to the control group. Taken together, treatment with 20-nm, negatively-charged ZnO NPs increased retinopathy, associated with local distribution of them in ocular lesions.
\end{abstract}

Key words: Zinc oxide, Nanoparticles, Ocular toxicity, Micro-CT, Histopathology

\section{INTRODUCTION}

Although nanoparticles (NPs) can be applied in several fields, including cosmetics, therapeutics and diagnostics, information about risk and safety evaluations of them is not sufficient to ensure their safety for human health (1). In particular, these NPs are often considerably more reactive than their bulk material counterparts as a result of their larger surface area; indeed, due to their size, NPs often exhibit unique

Correspondence to: Jin Seok Kang, Department of Biomedical Laboratory Science, Namseoul University, 91 Daehak-ro, Seonghwan-eup, Sebuk-gu, Cheonan 331-707, Korea

E-mail: kang@nsu.ac.kr

This is an Open-Access article distributed under the terms of the Creative Commons Attribution Non-Commercial License (http:// creativecommons.org/licenses/by-nc/3.0) which permits unrestricted non-commercial use, distribution, and reproduction in any medium, provided the original work is properly cited. physical/chemical properties and can impart enhancements to engineered materials, including better magnetic properties, improved electrical and optical activity, and increased structural integrity (2).

Zinc oxide $(\mathrm{ZnO})$ NPs are used widely in cosmetics such as sunscreens, foot care, ointments, and over-the-counter topical products and coatings (e.g., ultraviolet protection, fungicide in paints), electronic devices, and catalysts. $\mathrm{ZnO}$ NPs showed systemic distribution and the target organs included liver, spleen, lung, and kidney, and, in some cases, the heart (3). Despite the widespread use of ZnO NPs, the safety of this compound for humans is remains unclear.

Previous study showed that a 90-day treatment of $20 \mathrm{~nm}$, negatively charged $\mathrm{ZnO}$ NPs induced main changes in the pancreas, stomach and eyes (4). Among these, pathological examination of eyes represented the retinal atrophy. Although a recent in vitro study reported that $\mathrm{ZnO}$ NPs reduced the mitochondrial membrane potential, and increased the pro- 
duction of reactive oxygen species (ROS) in retinal ganglion cells (5), the related mechanisms of eye toxicity have not been clarified in vivo.

To assess the direct evidence of retinal atrophy in eyes, image-based technologies could be possible to identify of chemical(s). Several imaging technologies, such as microcomputed tomography (micro-CT), magnetic resonance imaging (MRI), optical tomography, fluorescence molecular tomographic imaging could be useful for this approach. Using image-based technologies, identification and optimization of chemical could be possible (6). Micro-CT have allowed scientists to capture detailed anatomical images and to identify foreign particles and to monitor the progression of disease in small animals (7). We tested the hypothesis that micro-CT analysis provided a readout of the potential location of $\mathrm{ZnO}$ NPs in eyes of rats. We aimed to investigate the detection of $\mathrm{ZnO}$ NPs in eyes of rats treated with $\mathrm{ZnO}$ NPs using micro-CT, to identify of zinc using X-ray fluorescence spectrometery (XFS) and energy dispersive Xray spectroscopy (EDS) and to confirm the eye lesions by conventional histopathological examination.

\section{MATERIALS AND METHODS}

Preparation of ZnO. ZnO NPs (ultra fine zinc oxide ZnO-310) were purchased from Sumitomo Osaka Cement Co., Ltd. (Osaka, Japan). The crystalline structure and size of $\mathrm{ZnO}$ NPs were analyzed; the mean diameter was $29 \pm 3$ $\mathrm{nm}$ in deionized water.

The cyrstalline structure and the size of $\mathrm{ZnO}$ NPs was analyzed by X-ray diffraction (XRD) and fourier transform inrafred (FT-IR) and the average diameter was $29 \pm 3 \mathrm{~nm}$ in deionized water. The surface charge modification was performed by using sodium citrate to add topical negative charges to the ZnO NPs. The HEPES buffer solution was first adjusted to $\mathrm{pH} 7$ using $1 \mathrm{M} \mathrm{Na}_{2} \mathrm{CO}_{3}$, and then sodium citrate was added to the HEPES buffer to produce HEPES citrate buffer ( $2 \%$ citrate). Next the ZnO NPs were suspended in the HEPES-citrate buffer for chemical modification, as previously reported (4). Then they were weighed and resuspended in HEPES-citrate buffer solution to yield a treatment dose NP solution. Preparation of freshly modified $\mathrm{ZnO}$ NPs for use was done daily over the course of the 90-day study.

The stability and homogeneity of the resultant $\mathrm{ZnO}$ NPs were confirmed using method validation and verification of the formulation concentration according to protocols established by the Korea Testing and Research Institute. The concentration was measured on days 1,45 and 90 , just prior to administration to the rats.

Test animals and experimental system. Five-weekold male and female, specific-pathogen free (SPF) SpragueDawley rats were purchased from Orient Bio, Inc. (Seongnam-si, Korea) and acclimated for 7 days prior to initiation of the study. During the acclimation and experimental periods, the rats were housed in wire cages (maximum of two rats per cage) in a room with controlled temperature $\left(22 \pm 3^{\circ} \mathrm{C}\right)$ and humidity $(50 \pm 20 \%)$ and a 12 $\mathrm{hr}$ light/dark cycle. The rats were fed a gamma ray-irradiated rodent diet (Cargill Agri Purina Korea Inc., Pyungtaek Seongnam Kyunggi-do, Korea) and filtered water ad libitum.

Rats were divided into four groups: G1 as vehicle control $(\mathrm{n}=10), \mathrm{G} 2$ as 20-nm, negatively-charged ZnO NPs treated for 90 days $(\mathrm{n}=10), \mathrm{G} 3$ as a vehicle control recovery group $(\mathrm{n}=5)$ and $\mathrm{G} 4$ as $\mathrm{ZnO}$ NPs treated for 90 days plus a 14day recovery period $(n=5)$. Ten eye samples per group were taken from the animals treated with vehicle (G1) and those treated with $500 \mathrm{mg} / \mathrm{kg} \mathrm{ZnO}$ NPs (G2) for 90 days. In addition, five eye samples per group were taken from the animals treated with vehicle (G3) and those treated with $500 \mathrm{mg} / \mathrm{kg} \mathrm{ZnO} \mathrm{NPs} \mathrm{(G4)} \mathrm{for} 90$ days plus a 14-day recovery period. This study was conducted according to the OECD Principles of Good Laboratory Practice and approved by the Institutional Animal Care and Use Committee.

Histopathology. Histological examinations were performed on the eyes from all animals. The eyes were fixed in $10 \%$ neutral phosphate-buffered formalin, embedded in paraffin, and sectioned to a thickness of $4 \mu \mathrm{m}$. For morphological examination, the sections were first deparaffinized and rehydrated. After rinsing with distilled water for $5 \mathrm{~min}$, the sections were stained with hematoxylin for $10 \mathrm{~min}$, rinsed with distilled water for $20 \mathrm{~min}$ and stained with eosin for 3 min. Finally, the sections were dehydrated in an ascending series of ethanol, cleared by xylene, and cover-slipped with mounting medium.

Immunohistochemistry. The sections were dewaxed in xylene, hydrated using a graded ethanol series and boiled in sodium citrate buffer $(\mathrm{pH}$ 6.0) in an autoclave for $20 \mathrm{~min}$. Next they were treated sequentially with $0.3 \%$ hydrogen peroxide, blocking buffer containing horse serum, and an antibody to neuronal nuclei (NeuN) (ab104225; Abcam, Cambridge, UK; diluted 1:500) for $1 \mathrm{hr}$. The sections were incubated with HRP-polymer (MRT621; Biocare Medical, Concord, CA). The immune complexes were visualized using 3,3'-diaminobenzidine tetrahydrochloride as a chromogen. The sections were counterstained with Mayer's hematoxylin to facilitate examination under a light microscope.

Micro-CT analysis. A Skyscan Desktop Micro-CT 1172 (Aartselaar, Belgium) instrument, with a source voltage of $60 \mathrm{kV}$, and current of $167 \mu \mathrm{A}$, was used to acquire X-ray radiographs. The specimens were attached to a stage that rotated $360^{\circ}$ with images acquired at $0.7^{\circ}$ intervals. After scanning, cross-sectional slices were reconstructed and each scan result was reconstructed using the 0.010 0.067 threshold values to distinguish bone and air. Three-dimensional 
analysis was performed using the Skyscan software, CTAn. The parameters of bone mass and micro-architecture, including the deposition and distribution of foreign materials in the eyes of rats treated with $\mathrm{ZnO}$ NPs, were evaluated using the built-in micro-CT software.

XFS and EDS. Air-dried filter sections were mounted on specimen stubs with self-adhesive carbon pads. The speci-
(A)

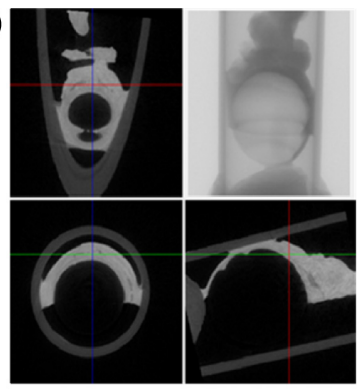

(C)

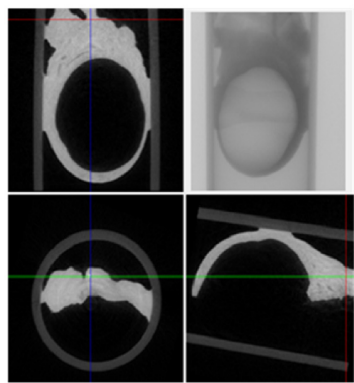

(B)

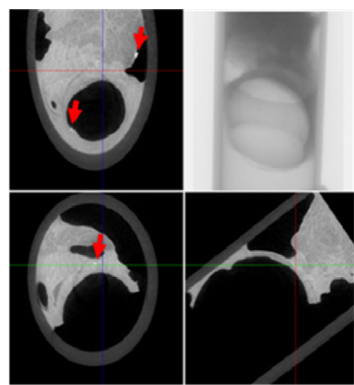

(D)

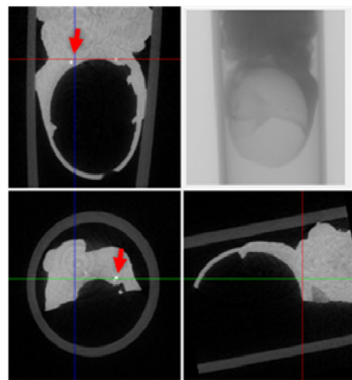

Fig. 1. Analysis of eyeball on $2 \mathrm{D}$ image of micro-CT. $2 \mathrm{D}$ image (coronal, transaxial, sagittal images); X-ray image. (A) vehicle treated groups for 90 days; (B) ZnO NPs treated groups for 90 days; (C) vehicle treated groups for 90 days plus a 14-day recovery period; (D) ZnO NPs treated groups for 90 days plus a 14day recovery period. Note observation of deposition and distribution of foreign materials (red arrow) in eye of rat treated with ZnO NPs.

(A) $[\mathrm{cps}]$

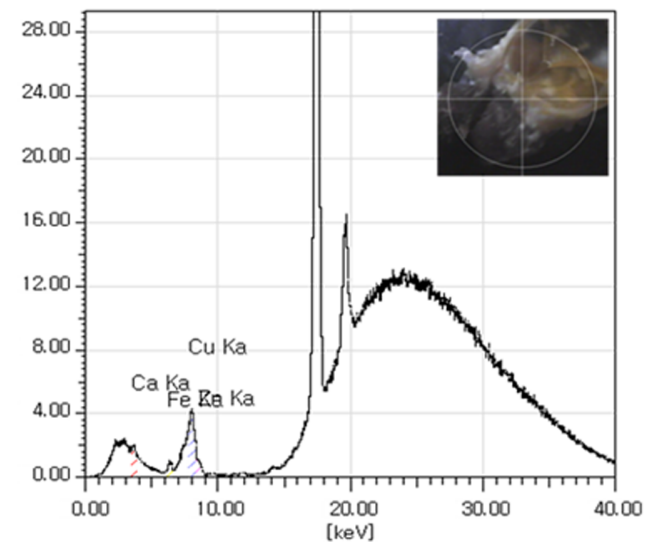

mens were coated with an electron conductive layer of carbon and evaluated using a JEOL electron microscope (JSM$7401 F$, Japan). The presence of zinc was assessed by XFS to determine zinc elemental composition and EDS to show the chemical characterization of samples.

\section{RESULTS}

Micro-CT analysis. Micro-CT analysis was conducted to examine the deposition and distribution of foreign materials in the eyes of rats treated with $\mathrm{ZnO}$ NPs (G2), com-
(A)

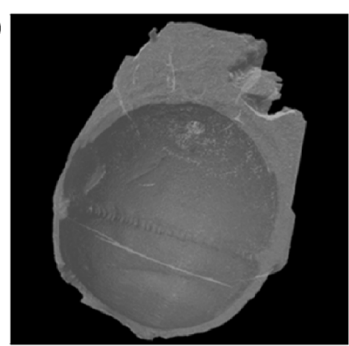

(C)

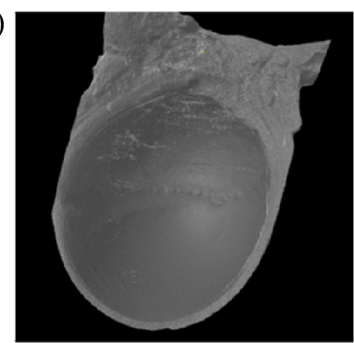

(B)

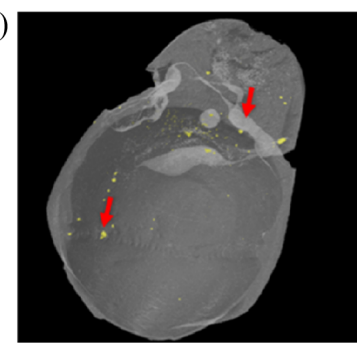

(D)

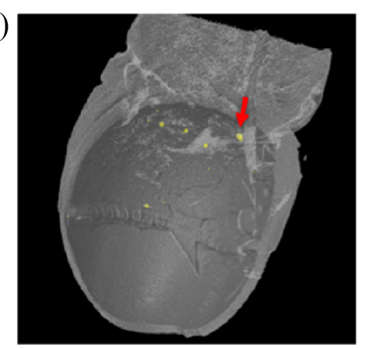

Fig. 2. Analysis of eyeball on $3 D$ image of micro-CT. (A) vehicle treated groups for 90 days; (B) ZnO NPs treated groups for 90 days; (C) vehicle treated groups for 90 days plus a 14-day recovery period; (D) ZnO NPs treated groups for 90 days plus a 14-day recovery period. Note observation of deposition and distribution of foreign materials (red arrow) in eye of rat treated with $\mathrm{ZnO}$ NPs.

(B)

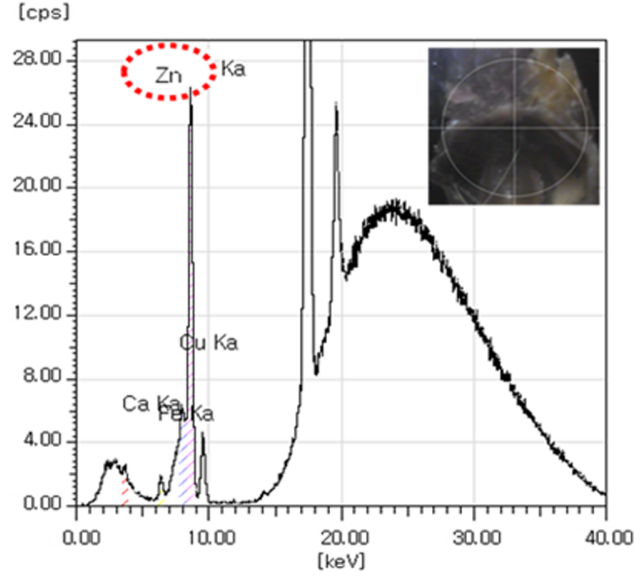

Fig. 3. X-Ray fluorescence spectroscopy of in the eyes of rats. (A) vehicle treated groups for 90 days; (B) ZnO NPs treated groups for 90 days. Note the $\mathrm{Zn}$ signal is appeared in the eye of rat treated with ZnO NPs, whereas not in the eye of vehicle-treated animal. 
pared to those treated with vehicle (G1) for 90 days. In the micro-CT 2D image analysis, the control animals showed no foreign materials (Fig. 1A). However, particles deposition was observed in $\mathrm{ZnO}$ NPs treatment groups (Fig. 1B). And the control animals treated with vehicle for 90 days

(A)

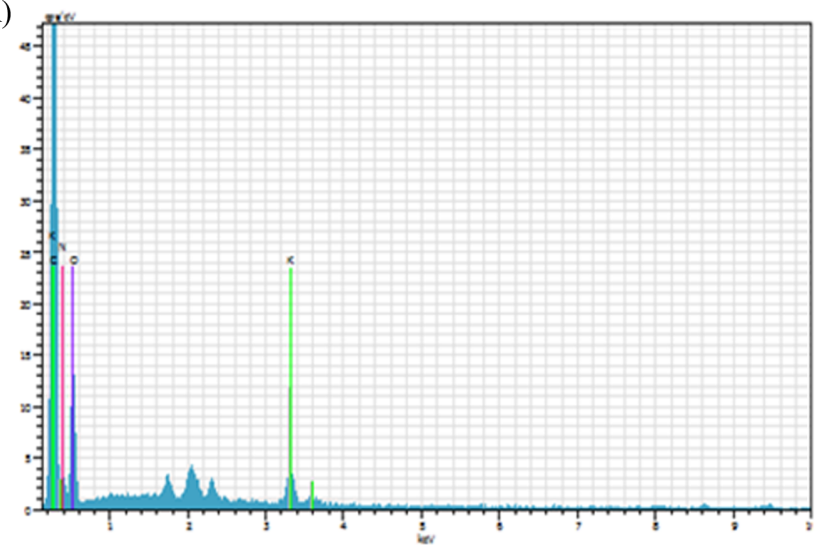

plus 14-day recovery (G3) showed no foreign materials (Fig. 1C). Particles deposition was also detected in the eyes of animals treated with $\mathrm{ZnO}$ NPs for 90 days plus 14-day recovery (G4) (Fig. 1D).

Using micro-CT 3D image analysis, the animals treated

(B)

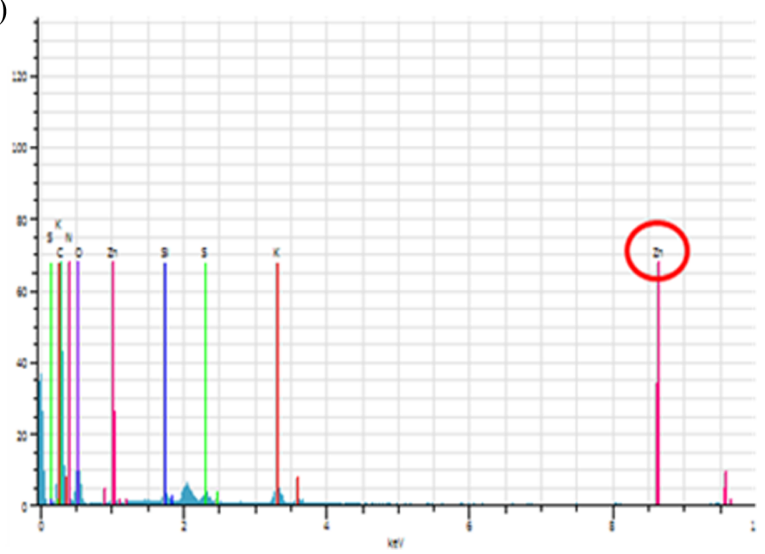

Fig. 4. Energy dispersive spectometery in the eyes of rats. (A) vehicle treated groups for 90 days; (B) ZnO NPs treated groups for 90 days. Note that $\mathrm{Zn}$ signal is appeared in the eye of rat treated with ZnO NPs, whereas not in the eye of vehicle-treated animal.
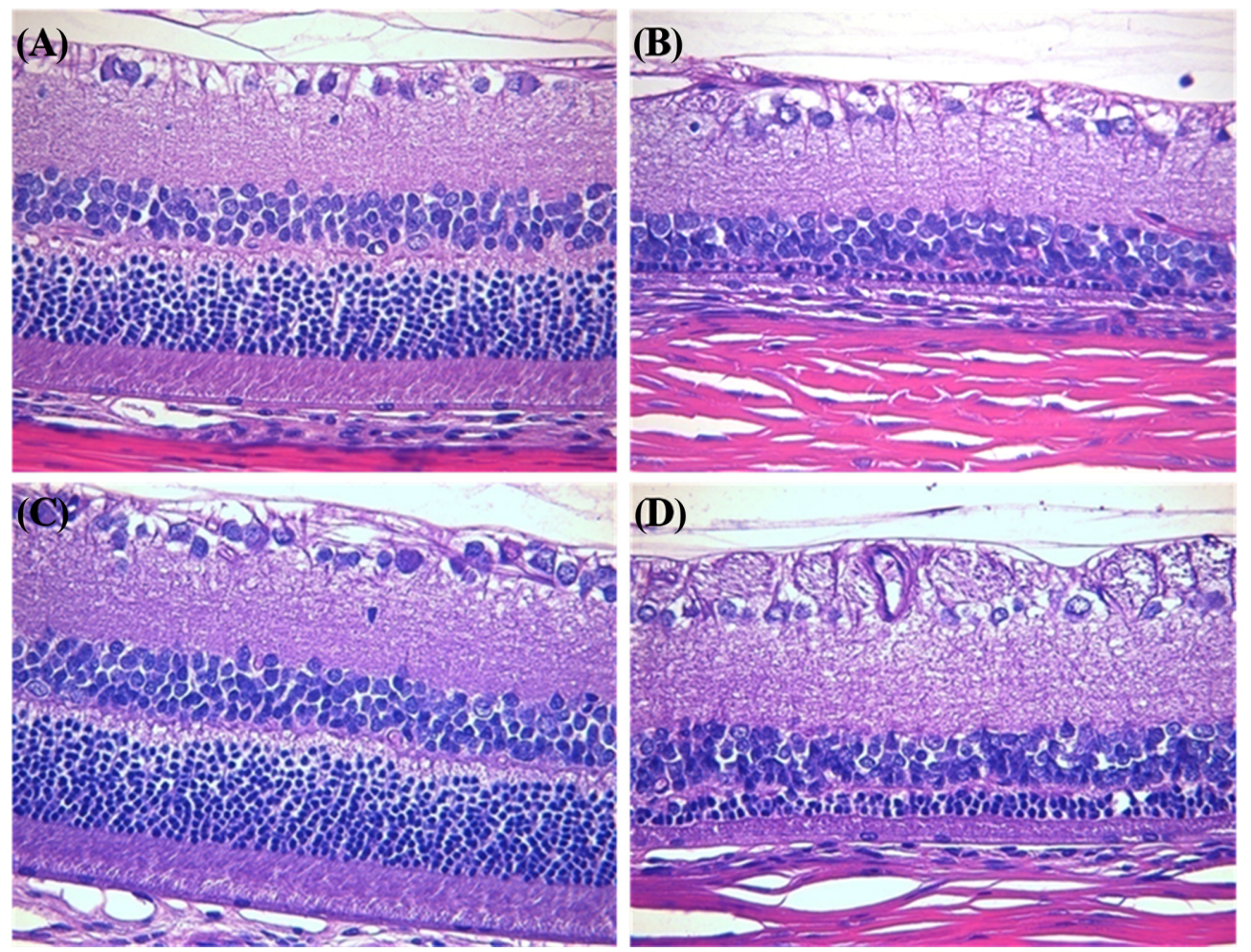

Fig. 5. Histopathological examination of retinopathy in the eyes of rats treated with zinc oxide nanoparticles (ZnO NPs). (A) vehicle treated groups for 90 days; (B) ZnO NPs treated groups for 90 days; (C) vehicle treated groups for 90 days plus a 14-day recovery period; (D) ZnO NPs treated groups for 90 days plus a 14-day recovery period. Note the normal microscopic structure of the eye of rat from control group. However, retinal atrophy is remarkable in the eye of rat treated with ZnO NPs for 90 days. Furthermore, this is still remaining in the eye of rat from ZnO NPs treatment and 14-day recovery group. Hematoxylin \& eosin staining of paraffin embedded sections from the eye from rat, Magnification, $\times 200$. 

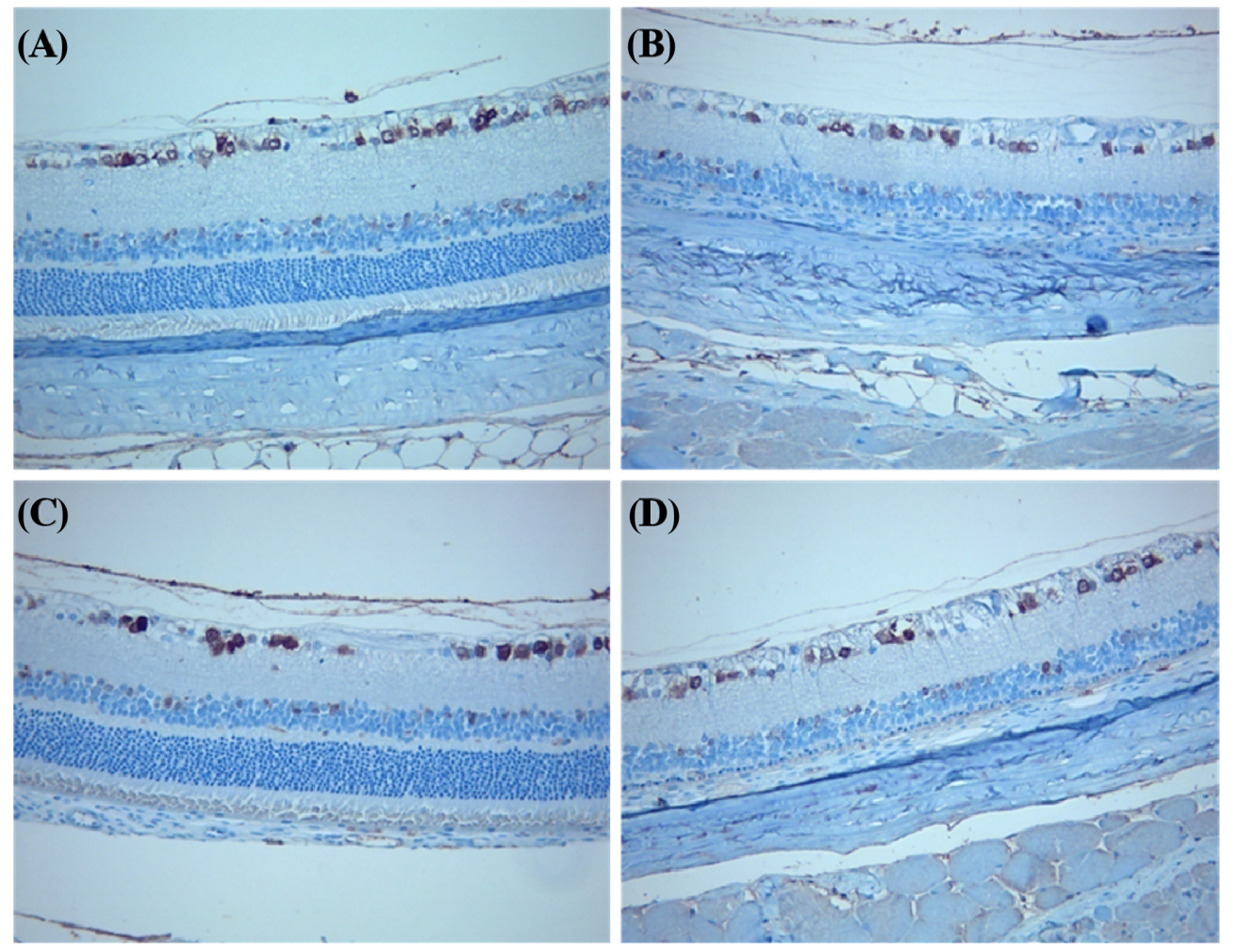

Fig. 6. Immunohistochemical expression of neuronal nuclei (NeuN) in the retina. (A) vehicle treated groups for 90 days; (B) ZnO NPs treated groups for 90 days; (C) vehicle treated groups for 90 days plus a 14-day recovery period; (D) ZnO NPs treated groups for 90 days plus a 14-day recovery period. Note the decreased of NeuN positive cells in retina of rat treated with $500 \mathrm{mg} / \mathrm{kg} \mathrm{ZnO} \mathrm{NPs} \mathrm{for} 90$ days compared to control. And this is still remaining in the eye of rat from ZnO NPs $500 \mathrm{mg} / \mathrm{kg}$ treatment and 14-day recovery group compared to age-matched control. Immunostainig of NeuN, Magnification, $\times 200$.

with vehicle (G1) for 90 days and 90 days plus 14-day recovery (G3) showed no foreign materials (Fig. 2A and $2 \mathrm{C}$, respectively). Conversely, particles were deposited in the eye ball and muscle and surrounding tissues of animals treated with $\mathrm{ZnO}$ NPs (G2) for 90 days and 90 days plus 14-day recovery (G4) (Fig. 2B and 2D, respectively).

XFS and EDS. XFS results showed $\mathrm{Zn}$ signal in the eyes of rats treated with ZnO NPs, but not in the eyes of control animals (Fig. 3). EDS showed $\mathrm{Zn}$ signal in the eyes of rats treated with $\mathrm{ZnO} N$ Ps, but not in the eyes of control animals (Fig. 4).

Histopathology. We observed test article-related ophthalmological alterations in the eyes of rats treated with $\mathrm{ZnO}$ NPs. Retina in vehicle control (G1) showed normal structure (Fig. 5A) and retinal atrophy between the inner nuclear layer and the outer nuclear layer was identified in the animals treated with $\mathrm{ZnO}$ NPs for 90 days (G2) (Fig. 5B). And retina in a vehicle control recovery group (G3) showed normal findings (Fig. 5C). However, retinal atrophy was also observed in the animals treated with $\mathrm{ZnO}$ NPs for 90 days plus 14-day recovery (G4) (Fig. 5D).
Immunohistochemistry. Treatment with ZnO NPs also resulted in retinal neuronal damage, as confirmed by NeuN immunostaining. NeuN expression was reduced in neurons of the ganglion cell layer of animals treated with $\mathrm{ZnO}$ NPs (G2) compared to those treated with vehicle (G1) for 90 days. NeuN expression was also reduced in retinal ganglion cells of the animals treated with ZnO NPs (G4) compared to those from age-matched control (G3) (Fig. 6).

\section{DISCUSSION}

The current findings demonstrated that continuous treatment with 20-nm, negatively-charged ZnO NPs increased retinopathy in rats. Histopathological analysis of the eyes of rats treated with $\mathrm{ZnO}$ NPs indicated retinopathy, and the location of the particles was confirmed by micro-CT, XFS and EDS analyses. Therefore, the ocular toxicity of $\mathrm{ZnO}$ NPs could be associated with their local distribution in ocular lesions.

Despite the previous studies on the toxicity of $\mathrm{ZnO} \mathrm{NPs}$, the molecular mechanism underlying their in vivo toxicity remains unclear. In this study, retinal atrophy between the inner and outer nuclear layers was identified in the animals 
treated with $\mathrm{ZnO}$ NPs. However, there was no inflammatory cell infiltration in those lesions. It has been reported that high amounts of zinc were contained in the retina and zinc localization in the photoreceptors was varied in dark and light, suggesting a role for zinc in a light-regulated process (8). And zinc staining was stronger in the outer retina, mainly in the outer plexiform layers and the outer limiting membrane (9). It has been reported that endogenous zinc contributed to retinal cell death after ischemia in cultured rat retinal cells (10). Exposure to white fluorescent light induced a marked increase in $\mathrm{Zn}$ ion staining, particularly in the superior outer nuclear layer and retinal pigment epithelial cells of dark-maintained Sprague-Dawley albino rats (11). Indeed, potassium also increased release of $\mathrm{Zn}$ ions from the rat retina, mainly in the outer plexiform layers and photoreceptor inner segment regions (12). Taken together, these findings demonstrated that the eye is extremely sensitive to $\mathrm{Zn}$, and exposure can cause retinal damage. As no inflammatory cell infiltration was observed in the retina in current study, this retinopathy may be induced by apoptosis of retinal cells.

In this study, NeuN expression was decreased in neurons of the ganglion cell layer of animals treated with $\mathrm{ZnO}$ NPs. A previous study reported that NeuN was present in most neuronal cell types of all vertebrates and its distribution was apparently restricted to neuronal nuclei, perikarya, and some proximal neuronal processes (13). It appeared that $\mathrm{ZnO}$ NPs induced the death of neurons in eyes. Another study reported that the production of large amounts of ROS and high oxygen consumption were major factors in many retinal diseases (14). Generally, NPs of various sizes and chemical compositions can lodge in mitochondria, possibly resulting in disruption of the mitochondrial electron transport chain (15). Moreover, the size, shape, surface chemistry, and degree of aggregation of NPs influenced the production of free radicals and subsequent oxidative stress (16), and have been associated with the content of redox cycling organic chemicals and their ability to damage mitochondria (17). A recent study suggested that $\mathrm{ZnO}$ nanoparticle-induced toxicity via ROS overproduction would trigger endoplasmic reticulum stress, leading to retinal ganglion cell damage and finally inducing apoptosis/necrosis, and the overexpression of caspase-12 might be involved in death of retinal ganglion cells (5). Furthermore, many NPs have been found to cause genotoxic responses, such as chromosomal fragmentation, DNA strand breakage, point mutations, oxidative DNA adducts and alterations in gene expression profiles (18). Moreover, high amounts of ROS play a role in a number of disease states, including cancer, ischemia, and failures in immunity (19). Indeed, concentration- and time-dependent cytotoxicity and an increase in DNA and cytogenetic damage have been associated with high zinc oxide concentrations (20).

In the current study, $\mathrm{ZnO}$ NPs were administred to rats via the oral route; however, the route by which they are translocated into the eye is unclear. It has been reported that small sized NPs could facilitate uptake into cells and transcytosis across epithelial and endothelial cells into the blood and lymph circulation to reach potentially sensitive target sites, and even the central nervous system and ganglia, via translocation along the axons and dendrites of neurons (21). The uptake of NPs by cells involves binding with the cell membrane followed by internalization; the size, shape, surface chemistry, and degree of aggregation of NPs affect the production of free radicals and resultant oxidative stress (16). In the current study, ZnO NPs might have spread into the blood and lymph circulation, and thence into the eyes. Further studies are required to investigate the pathway of nanoparticle translocation into eyes.

The XFS and EDS analyses in this study detected zinc signals in the ZnO NPs treatment group, but not in the control group. Whether the increased intracellular $\mathrm{Zn}$ ion levels were due to the NPs being taken up by cells or their dissolution in medium remain unclear (3). Dissolution, translocation, and deposition have been demonstrated to play key roles in the fate and effects of conventional fibers and particles (22), and rapid dissolution of $\mathrm{ZnO}$ nanoparticles in fresh water medium $(\mathrm{pH} 7.6)$, which have a saturation solubility in the milligram per liter range, similar to that of bulk $\mathrm{ZnO}$ (23). Based on the current findings, it is unclear whether the retinopathy was caused by $\mathrm{ZnO}$ NPs themselves or $\mathrm{Zn}$ ions. Further studies should investigate the physiochemical properties of ZnO NPs in eyes.

In this study, we applied micro-CT analysis to investigate the location of $\mathrm{ZnO}$ NPs in eyes. However, the sampling process might have induced technical translocation of $\mathrm{ZnO}$ NPs in the eyes. In vivo imaging allows the assessment of toxicity and monitoring of the response of specific organs to nanoparticles. To assess a direct role in eye toxicity, use of a combination of advanced imaging with three-dimensional systems and intravital animal models would provide more informative and disease-relevant information (6). Intravital microscopy could facilitate measurement of the functionality and dynamics of individual regulators of migration (24). Furthermore, imaging of living animals reduces the number of animals required per experiment and improves the statistical power, as each animal can function as its own control over time (25). Further studies should involve monitoring of living animals treated with $\mathrm{ZnO}$ NPs using in situ imaging tools, such as magnetic resonance imaging and ultrasound, as well as micro-CT.

Therefore, treatment with 20-nm, negatively-charged $\mathrm{ZnO}$ NPs increased retinopathy associated with local distribution of them in ocular lesions.

\section{ACKNOWLEDGEMENTS}

This study was supported by a grant (13181MFDS605) 
from the Korean Ministry of Food and Drug Safety in 2013.

\section{CONFLICT OF INTEREST}

Authors declare that there are no conflicts of interest.

\section{REFERENCES}

1. Thomas, K., Aguar, P., Kawasaki, H., Morris, J., Nakanishi, J. and Savage, N. (2006) Research strategies for safety evaluation of nanomaterials, part VIII: International efforts to develop risk-based safety evaluations for nanomaterials. Toxicol. Sci., 92, 23-32.

2. Thomas, K. and Sayre, P. (2005) Research strategies for safety evaluation of nanomaterials, Part I: evaluating the human health implications of exposure to nanoscale materials. Toxicol. Sci., 87, 316-321.

3. Vandebriel, R.J. and De Jong, W.H. (2012) A review of mammalian toxicity of $\mathrm{ZnO}$ nanoparticles. Nanotechnol. Sci. Appl., 5, 61-71.

4. Park, H.S., Shin, S.S., Meang, E.H., Hong, J.S., Park, J.I., Kim, S.H., Koh, S.B., Lee, S.Y., Jang, D.H., Lee, J.Y., Sun, Y.S., Kang, J.S., Kim, Y.R., Kim, M.K., Jeong, J., Lee, J.K., Son, W.C. and Park, J.H. (2014) A 90-day study of subchronic oral toxicity of $20 \mathrm{~nm}$, negatively charged zinc oxide nanoparticles in Sprague Dawley rats. Int. J. Nanomed., 9 Suppl 2, 79-92.

5. Guo, D., Bi, H., Liu, B., Wu, Q., Wang, D. and Cui, Y. (2013) Reactive oxygen species-induced cytotoxic effects of zinc oxide nanoparticles in rat retinal ganglion cells. Toxicol. In Vitro, 27, 731-738.

6. Conway, J.R., Carragher, N.O. and Timpson, P. (2014) Developments in preclinical cancer imaging: innovating the discovery of therapeutics. Nat. Rev. Cancer, 14, 314-328.

7. Schambach, S.J., Bag, S., Schilling, L., Groden, C. and Brockmann, M.A. (2010) Application of micro-CT in small animal imaging. Methods, 50, 2-13.

8. Ugarte, M. and Osborne, N.N. (2001) Zinc in the retina. Prog. Neurobiol., 64, 219-249.

9. Lee, S.C., Zhong, Y.M., Li, R.X., Yu, Z. and Yang, X.L. (2008) Localization of zinc in the outer retina of carp: a lightand electron-microscopic study. Synapse, 62, 352-357.

10. Yoo, M.H., Lee, J.Y., Lee, S.E., Koh, J.Y. and Yoon, Y.H. (2004) Protection by pyruvate of rat retinal cells against zinc toxicity in vitro, and pressure-induced ischemia in vivo. Invest. Ophthalmol. Visual Sci., 45, 1523-1530.

11. Sheline, C.T., Zhou, Y. and Bai, S. (2010) Light-induced photoreceptor and RPE degeneration involve zinc toxicity and are attenuated by pyruvate, nicotinamide, or cyclic light. Mol. Vision, 16, 2639-2652.
12. Redenti, S. and Chappell, R.L. (2005) Neuroimaging of zinc released by depolarization of rat retinal cells. Vision Res., 45, 3520-3525.

13. Mullen, R.J., Buck, C.R. and Smith, A.M. (1992) NeuN, a neuronal specific nuclear protein in vertebrates. Development, 116, 201-211.

14. Wenzel, A., Grimm, C., Samardzija, M. and Remé, C.E. (2005) Molecular mechanisms of light-induced photoreceptor apoptosis and neuroprotection for retinal degeneration. Prog. Retinal Eye Res., 24, 275-306.

15. Nel, A., Xia, T., Madler, L. and Li, N. (2006) Toxic potential of materials at the nanolevel. Science, 311, 622-627.

16. Aillon, K.L., Xie, Y., El-Gendy, N., Berkland, C.J. and Forrest, M.L. (2009) Effects of nanomaterial physicochemical properties on in vivo toxicity. Adv. Drug Delivery Rev., 61, 457-466.

17. Li, N., Sioutas, C., Cho, A., Schmitz, D., Misra, C., Sempf, J., Wang, M., Oberley, T., Froines, J. and Nel, A. (2003) Ultrafine particulate pollutants induce oxidative stress and mitochondrial damage. Environ. Health Perspect., 111, 455-460.

18. Singh, N., Manshian, B., Jenkins, G.J., Griffiths, S.M., Williams, P.M., Maffeis, T.G., Wright, C.J. and Doak, S.H. (2009) NanoGenotoxicology: the DNA damaging potential of engineered nanomaterials. Biomaterials, 30, 3891-3914.

19. Matés, J.M., Pérez-Gómez, C. and Nunez de Castro, I. (1999) Antioxidant enzymes and human diseases. Clin. Biochem., 32, 595-603.

20. Osman, I.F., Baumgartner, A., Cemeli, E., Fletcher, J.N. and Anderson, D. (2010) Genotoxicity and cytotoxicity of zinc oxide and titanium dioxide in HEp-2 cells. Nanomedicine (London), 5, 1193-1203.

21. Oberdörster, G., Oberdörster, E. and Oberdörster, J. (2005) Nanotoxicology: an emerging discipline evolving from studies of ultrafine particles. Environ. Health Perspect., 113, 823839.

22. Borm, P., Klaessig, F.C., Landry, T.D., Moudgil, B., Pauluhn, J., Thomas, K., Trottier, R. and Wood, S. (2006) Research strategies for safety evaluation of nanomaterials, part V: role of dissolution in biological fate and effects of nanoscale particles. Toxicol. Sci., 90, 23-32.

23. Franklin, N.M., Rogers, N.J., Apte, S.C., Batley, G.E., Gadd, G.E. and Casey, P.S. (2007) Comparative toxicity of nanoparticulate $\mathrm{ZnO}$, bulk $\mathrm{ZnO}$, and $\mathrm{ZnCl} 2$ to a freshwater microalga (Pseudokirchneriella subcapitata): the importance of particle solubility. Environ. Sci. Technol., 41, 8484-8490.

24. Ellenbroek, S.I. and van Rheenen, J. (2014) Imaging hallmarks of cancer in living mice. Nat. Rev. Cancer, 14, 406-418.

25. de Jong, M., Essers, J. and van Weerden, W.M. (2014) Imaging preclinical tumour models: improving translational power. Nat. Rev. Cancer, 14, 481-493. 